Review of Income and Wealth

Series 59, Number 4, December 2013

DOI: $10.1111 / \mathrm{j} .1475-4991.2012 .00521 . x$

\title{
NONSTATIONARITY AND STOCHASTIC STABILITY OF RELATIVE INCOME CLUBS
}

\author{
By Mahmoud A. El-Gamal
}

Rice University

AND

DEOCKHYUN RYU*

Chung-Ang University

\begin{abstract}
The recent literature on "convergence" of cross-country per capita incomes has been dominated by the two hypotheses of "global convergence" and "club-convergence," pertaining to limits of estimated income distribution dynamics. Utilizing a new measure of "stochastic stability," we establish two stylized facts regarding short- and medium-term distribution dynamics. The first is non-stationarity of transition dynamics, in the sense of changing transition kernels, and the second is emergence, disappearance, and re-emergence of a "stochastically stable" middle income group. This middle income group emerges as the gap between rich and poor clubs gets larger, and it changes the dynamics of transition to and from the rich and poor clubs, eventually narrowing the gap between the poor and rich as the middle club vanishes. Analyzing the stochastic stability of middle-income groups is thus a first step toward understanding higher-order dynamics of narrowing or widening of the gap between rich and poor countries.
\end{abstract}

JEL Codes: C4, O47

Keywords: convergence clubs, distributional dynamics, nonstationary dynamics, stochastic stability

\section{INTRODUCTION}

In recent decades characterized by increasing globalization, few issues have been debated more hotly than the issue of income gaps between rich and poor countries. The neoclassical growth model (with well-behaved aggregate production functions) suggests that the gap between rich and poor countries should be collapsing. This was formalized in the early empirical literature in terms of two types of "convergence hypotheses": absolute and conditional convergence. Early empirical results supported what came to be known as $\beta$-convergence: negative estimated coefficients for "initial income" variables in income growth regressions (Barro and Sala-i-Martin, 1992, 1995; Mankiw et al., 1992).

Comforting convergence results were soon challenged on both theoretical and empirical grounds. On theoretical grounds, endogenous growth models challenged the credibility of - and robustness to-classical assumptions of well-behaved

Note: We are grateful to Paul Johnson, Essie Maasoumi, Jeff Racine, two anonymous referees, and participants at the Texas Camp Econometrics for valuable comments.

*Correspondence to: Deockhyun Ryu, Associate Professor of School of Economics, Chung-Ang University, Seoul, Korea (dhryu@cau.ac.kr).

(C) 2012 The Authors

Review of Income and Wealth (C) 2012 International Association for Research in Income and Wealth 
aggregate production functions. With minor modifications of the Solow aggregate production function, non-convexities and poverty traps were generated, for example in threshold models of Azariadis and Drazen (1990) and Durlauf (1993), among others. Meanwhile, on the empirical front, simple cross-country growth regressions that lent support to the $\beta$-convergence hypothesis were replaced by panel-data, time-series (unit-root and co-integration) and distributional-dynamics analyses, especially after Phillips and Sul (2003) showed that $\beta$ - and $\sigma$-convergence do not obtain if economies converge at different rates. Durlauf et al. (2005) provided an excellent survey of earlier developments in the empirical literature.

The distributional-dynamics approach to studying income distribution dynamics has become more popular in recent years, starting with Quah (1993a, 1993b, 1995, 1996a, 1996b, 1997, 2001) and Durlauf (1993), followed by many papers, including Bianchi (1997), Desdoigts (1999), Johnson (2000, 2005), Bulli (2001), Fiaschi and Lavezzi (2003), Maasoumi et al. (2006), and Pittau et al. (2010), among others. Unlike the linear regression approach, the distributionaldynamics literature focused on richer non-linear dynamics and sample heterogeneity, as captured by probability transition kernels, often estimated non- or semi-parametrically, for the distributions of world income or growth rates. The primary conclusion of this massive literature has been rejection of the global convergence hypothesis, based on evidence of multi-modality or other measures of polarization in the limiting distribution of per capita incomes.

One may argue that even within the frameworks of global convergence, club convergence, etc., we care mostly about the relevance of current and past income dynamics and their effects on income-gaps between rich and poor countries in the short- to medium-term. In order to study short- and medium-term relative income dynamics, we propose a measure of stochastic stability representing the relative likelihood of any given country (at a particular relative income) getting relatively richer, relatively poorer, or staying the same. A stochastically stable point on the income distribution scale is a ranking with a "stochastic basin of attraction," whereby countries that are slightly richer are likely (probability $>0.5$ ) to get poorer, and those slightly poorer are likely to get richer. Our analysis of short- to mediumterm income-clubs is thus reduced to a statistical analysis of the number of such stochastically stable points; one for which we derive a formal statistical test.

The early income-distribution dynamics literature, for example Quah (1997), found strong evidence of bimodality of limiting income distributions. This result was presented as evidence of ongoing polarization of world income distributions, and vanishing middle-income groups. Those results were confirmed by further analyses, for example Bianchi (1997), Paap and van Dijk (1998), Fiaschi and Lavezzi (2003), Anderson (2004a, 2004b) and Maasoumi et al. (2006). However, Vollmer et al. (2012) and Pittau and Zelli (2006) have more recently presented evidence in favor of the presence of three peaks in the distribution of per capita GDP. In contrast, an earlier literature, including Kremer et al. (2001) and Sala-iMartin (2002a, 2002b) had conducted distribution-dynamics analyses wherein they found evidence of uni-modality of the limiting distribution. These earlier results were argued to have cast doubts on the robustness of club-convergence distribution dynamics results, and supported the earlier linear-model results of global convergence. 
Quah (2001) pointed out that the literature on distributional dynamics had until then relied mainly on ocular inspection of estimated ergodic measures, and lacked a satisfactory formal test of the club convergence hypothesis. Formal tests of multi-modality were developed by Bianchi (1997), Kremer et al. (2001), Anderson (2004a, 2004b), and Maasoumi et al. (2006). However, Bianchi's (1997) test focused on multi-modality of the static (cross-sectional) income distribution density. Hence, that test is relevant only to the case where cross-section income observations were drawn from the unique invariant (ergodic) measure.

Later, Anderson (2004a, 2004b) and Maasoumi et al. (2006) suggested direct tests of income polarization using stochastic dominance ranking. Anderson (2004a, 2004b) interpreted divergence or polarization in terms of patterns of relocation of distributional mass. Thus he conducted his test by investigating stochastic dominance ordering between rich and poor income clubs after separately identifying the two groups. Similarly, Maasoumi et al. (2006) quantified distribution distances between the OECD group and non-OECD group based on entropy measures, which they used to construct statistical tests of convergence. Both sets of studies find empirical support for the convergence clubs and polarization hypotheses. Pittau et al. (2010) use a mixture of densities approach to reach similar results, with nuanced analysis of movements within and between income clubs, providing further evidence that counting modes may not be sufficient to understanding club dynamics.

Most tests in the literature have focused on analysis of cross-sectional distributions during different time periods, whereas the Kremer et al. (2001) test was constructed as a test of one versus more modes in the limiting distribution. In our analysis, we suggest that the alternative to two income-clubs (rich and poor) need not be global convergence. Indeed, the existence of a stable middle-income group (a third mode) will prove to be important in our analysis in Section 5 for studying the probability of transitions between poor, middle, and high income status in the medium- to long-term. The existence of a stable middle-income group did not play any role in the earlier tests of multimodality based on stochastic dominance, since that literature had pre-identified only two distinct income groups.

The rest of this paper will proceed as follows. In Section 2, we establish empirical stylized facts regarding the periodic appearance, disappearance, and reappearance of a middle-income group, as well as non-stationarity of income distribution dynamics, and introduce our proposed measure of "stochastic stability" of relative income groups. (In Appendix A, we reinforce the intuition derived from Section 2 by deriving a dynamic version of Bianchi's (1997) test to a nonparametric estimate of the limiting distribution, which may also be interpreted as a continuous version of the Kremer et al. (2001) test that relied on ad hoc discretization of income groups. Our analysis of those modified tests reinforce our view that any test that is focused on "convergence" or "polarization" between the rich and poor is insufficient, since the process displays fundamental non-stationarity, and the existence of a middle income group can facilitate escape from a poverty trap, at least for some countries.) In Section 3, we derive the asymptotic distribution theory for our measure of stochastic stability, thus paving the road for a formal statistical test of the stability of multiple income-distribution clubs, which is presented in Section 4. We conclude the paper in Section 5 with discussions of 
the economic implications of our stylized facts and empirical results, including the probabilities of transition between various relative income clubs.

\section{Empirical Stylized Facts}

We begin by providing graphical evidence of two stylized facts in income distribution dynamics, which we later confirm through rigorous statistical analysis. The first stylized fact is periodic appearance, disappearance, and reappearance of a middle-income group, and the second is the periodic change (non-stationarity) in the transition kernel itself. The latter stylized fact suggests that analysis of medium-term dynamics may prove more fruitful than analyses of asymptotic convergence or lack thereof, thus motivating our analysis of periodic stochastic stability of various income groups.

The data we utilize in this paper is taken from the Summers and Heston Penn World Table Mark 7.0, available at http://pwt.econ.upenn.edu, which covers 109 countries over the period 1960 to 2009. Our measure of a country's position in the global income distribution is the most widely used measure of relative income, which we calculated using PPP-based per capita incomes of the various countries. As commonly done in this literature, we define relative income as a percentage share of total world income. Relative income $x_{i}$ is thus calculated as follows: $x_{i}=\left(X_{i} / \Sigma_{j} X_{j}\right) \times 100$, where $X_{i}$ is per capita GDP of a country.

We must mention at this juncture that a number of recent studies have suggested replacing national per capita GDPs as objects of investigation of income distribution dynamics with global individual incomes (Bourguignon and Morrison, 2002; Sala-i-Martin, 2002a, 2002b; Fischer, 2003). With the exception of Bourguignon and Morrison's (2002) results, this alternative object of investigation has provided support for a modified version of the classical convergence hypothesis, mainly by giving significant weight to China and India, which simultaneously account for one-third of the world population, and exhibit some of the fastest rates of per capita income growth in the world. Proponents of this alternative object of investigation criticized per capita GDP as an oversimplified measure that ignores within-country inequality effects (Bourguignon and Morrison, 2002). For instance, by population-weighting real per capita GDP, Fischer (2003) found evidence of classical $\beta$-convergence. Using kernel density estimates of within- and between-country income distributions, Sala-i-Martin (2002a, 2002b) concluded that world income inequality was on the decline, arguing that the twin-peaks found in earlier literature were in fact vanishing, and a world middle-class was emerging (Sala-i-Martin, 2002b, p. 14).

This alternative empirical literature notwithstanding, we have decided to limit our analysis to more traditional cross-country per capita GDP distribution dynamics for two main reasons. First, the bulk of the literature has continued to focus on that measure, and hence we use it for comparability of results. Second, the literature suggested that between-country income inequality effects dominate within-country effects (Bourguignon and Morrison, 2002, table 2; Sala-i-Martin, 2002a, p. 39). Indeed, our own analysis confirmed the relative insignificance of within-country effects, provided that the weights given to China and India are 

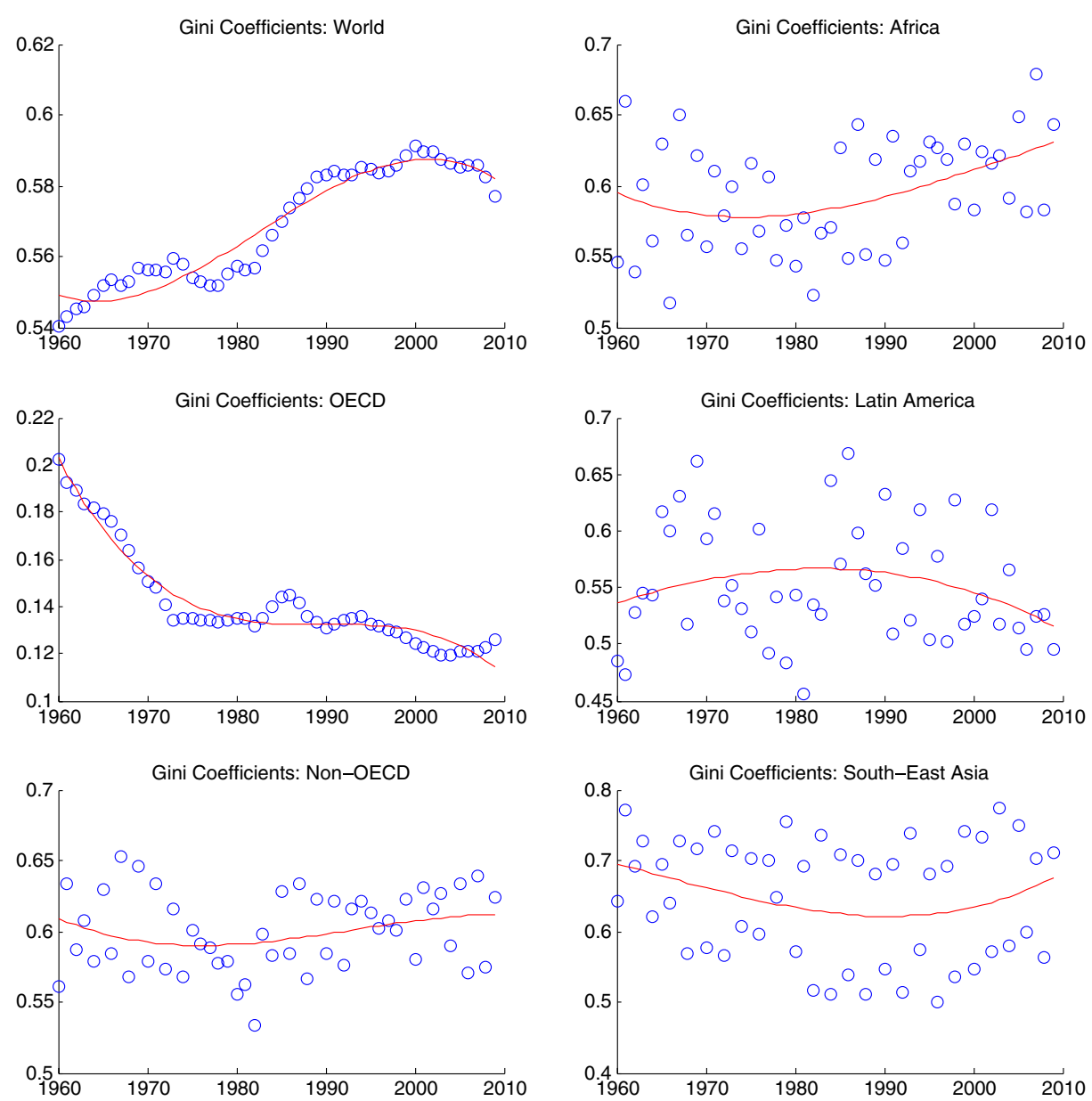

Figure 1. Annual Gini Coefficients for Various Subregions, with Cubic Trend

capped. ${ }^{1}$ When the weights given to China and India relative to the averagepopulation country in our sample were capped at 50 or less, the results reported in this paper do not change substantively if we consider within- as well as betweencountry effects. Nevertheless, as an anonymous referee has pointed out, the two types of estimates have different implications (differences across countries versus differences across world population) and the underlining analysis can have different goals (e.g. for policy implications the typical units of observation are individual countries, as we have used in this paper).

Our first stylized fact is graphically suggested by two different representations of the data. First, we plot time series of cross-country per capita GDP the Gini coefficients, computed, as usual as: $G=\sum_{i=1}^{n} \sum_{j=1}^{n}\left|x_{i}-x_{j}\right| /\left(2 n^{2} \mu\right)$, where $\mu$ is mean size. The first graph in Figure 1 shows that Gini coefficients for our entire sample

${ }^{1}$ Results reported in chapter 3 of Ryu (2004), using PWT Mark 6.1, are available upon request. 
(including developed and developing countries) trend upwards. On the other hand, the second graph in the left panel in Figure 1 shows that the Gini coefficient within the group of OECD countries is declining over time. Those two graphs support the hypothesis that world income distribution evolved in a polarized manner into rich and poor clubs. Further dividing the non-OECD group into three geographical sub-groups (graphs in the right panel in Figure 1), African, Latin American, and East and Southeast Asian, we find evidence of non-monotonicity. Those subgroups include Latin American and Asian countries that occupied a middleincome position for various sub-sample periods. The non-monotonicity of their Gini coefficients' time series suggests the possible emergence, disappearance, and reappearance of a middle income group. ${ }^{2}$

To investigate the emergence and stability of this middle-income group, we propose a measure of "stochastic stability," constructed as follows. We consider the median of any given country's relative income at time $t+1$ conditional on its relative income at time $t$, less that country's relative income at time $t$. In other words, denoting period $t$ relative income of a given country by $x_{t}$, we consider the measure:

$$
f(a)=\operatorname{median}\left(x_{t+1} \mid x_{t}=a\right)-a .
$$

A zero of this function occurs at any value $a$ such that a country with that relative income is equally likely to move up in relative income distribution as it is to move down. Plots of this measure are qualitatively similar to those used by Quah (1997) and Johnson (2000), to investigate income distribution dynamics graphically. However, as we shall see shortly, a distribution theory is relatively easy to develop for our proposed measure.

We now proceed to define the "stochastic stability" of a zero of our function $f($.). It is clear that whenever $f(a)$ is positive (negative), a country with relative income $a$ is likely (probability $>0.5$ ) to move up (down) in the income distribution. Consequently, a stochastically stable zero of $f($.) is defined as a point at which the function crosses the x-axis from above. For instance, Figure 2 illustrates a hypothetical function $f($.) with five zeros. Three of those zeros are obtained by crossing from above (C1, C3, and C5), and thus labeled "stochastically stable," in the sense that countries slightly to the left of those points are likely to move to the right, and vice versa. In contrast, $\mathrm{C} 2$ and $\mathrm{C} 4$, wherein the function crosses the $\mathrm{x}$-axis from below, are labeled "stochastically unstable." We may reinterpret Quah's asymptotic "twin peaks" hypothesis within the context of short- to medium-term dynamics as a function $f($.) with two stochastically stable zeros, whereas a function with three stochastically stable zeros would indicate that there are three clubs, the middle one being Sala-i-Martin's "emerging middle class." Notice in this regard that the function $f($.$) is derived directly from the transition kernel, and not merely$ from its hypothesized asymptotic limit.

${ }^{2}$ Further sub-dividing African countries into four regional groups (western, central, eastern, southern), we detect more within-group heterogeneity of Gini coefficients. Sala-i-Martin (2002a) has clearly made the case for the importance of understanding various income dynamics within Africa, as a tool for better understanding global income dynamics. 


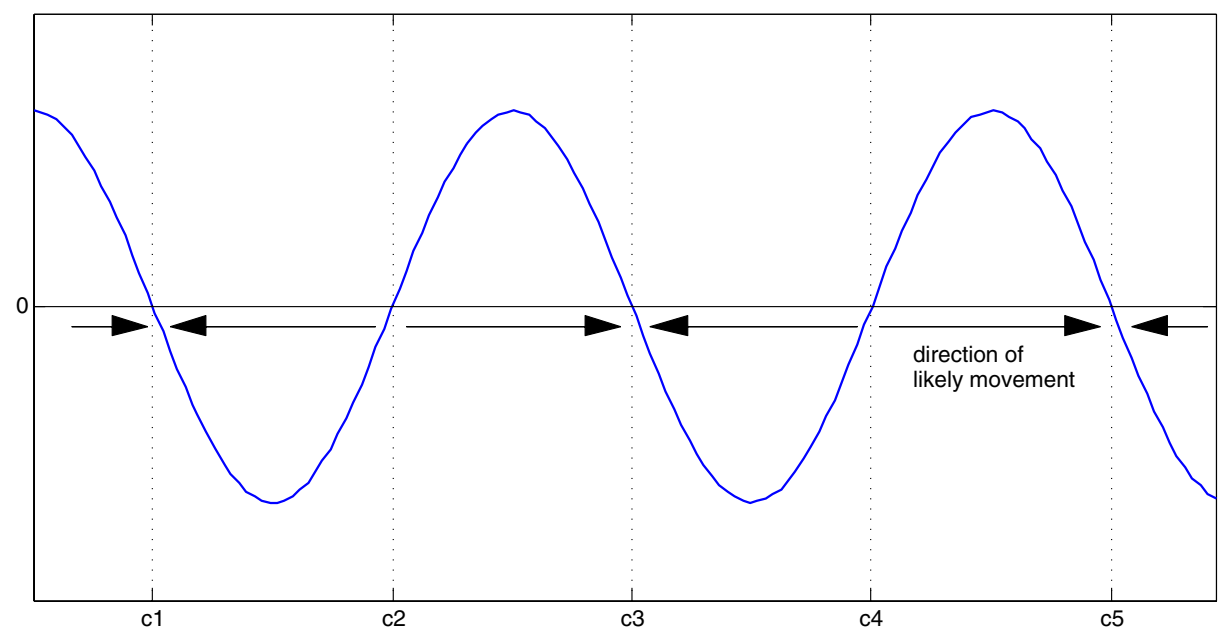

Figure 2. Stochastic Stability Diagram for Hypothetical Process with Five Zeros

Utilizing plots of our proposed function, we can establish both stylized facts mentioned at the beginning of this section. The panels in Figure 3 show plots of non-parametric estimates of the function $f($.) for each of the five decades 1960 2009. In Section 3, we shall define the proposed non-parametric method for estimating $f($.$) , and the asymptotic distribution of the resulting estimate, which will$ allow us to place pointwise confidence intervals around our estimate. Before constructing those confidence intervals, however, it seems compelling to conclude that there were two stable zeros in the 1960s, but three stable zeros during the 1970s and 1980s (not surprisingly, as the so-called "Asian Tigers" occupied the middle-income region during the latter period). The stable middle group was at a modest relative income level during the 1970s, but moved to a relatively high income in the 1980s. In the 1990s and the last decade, some of the emerging economies of the 1970s-80s, such as South Korea, have converged to the "rich club," while others have converged to poorer countries' per capita incomes. By the end of our 50-year sample, the stable middle-income club seems to have disappeared again. The same pattern holds if we divide the sample into three instead of five decade-long subperiods. ${ }^{3}$ The panels in Figure 4 show the function $f($.) for the sample subperiods 1960-75, 1976-91, and 1991-2009, respectively, again illustrating the appearance of the middle-income group (a third stable zero) during the middle subperiod and its disappearance in the last part of the sample.

To illustrate the transition dynamics for specific countries, we performed 5-means clustering for all countries in our sample for each of the five decades. Table 1 shows some interesting dynamics for eight countries. For each country and each decade, we show the country's relative income rank (higher rank means richer country), and cluster rank ( $1-5,1$ denoting the poorest club and 5 the richest).

\footnotetext{
${ }^{3}$ This robustness check was suggested by an anonymous referee.
} 

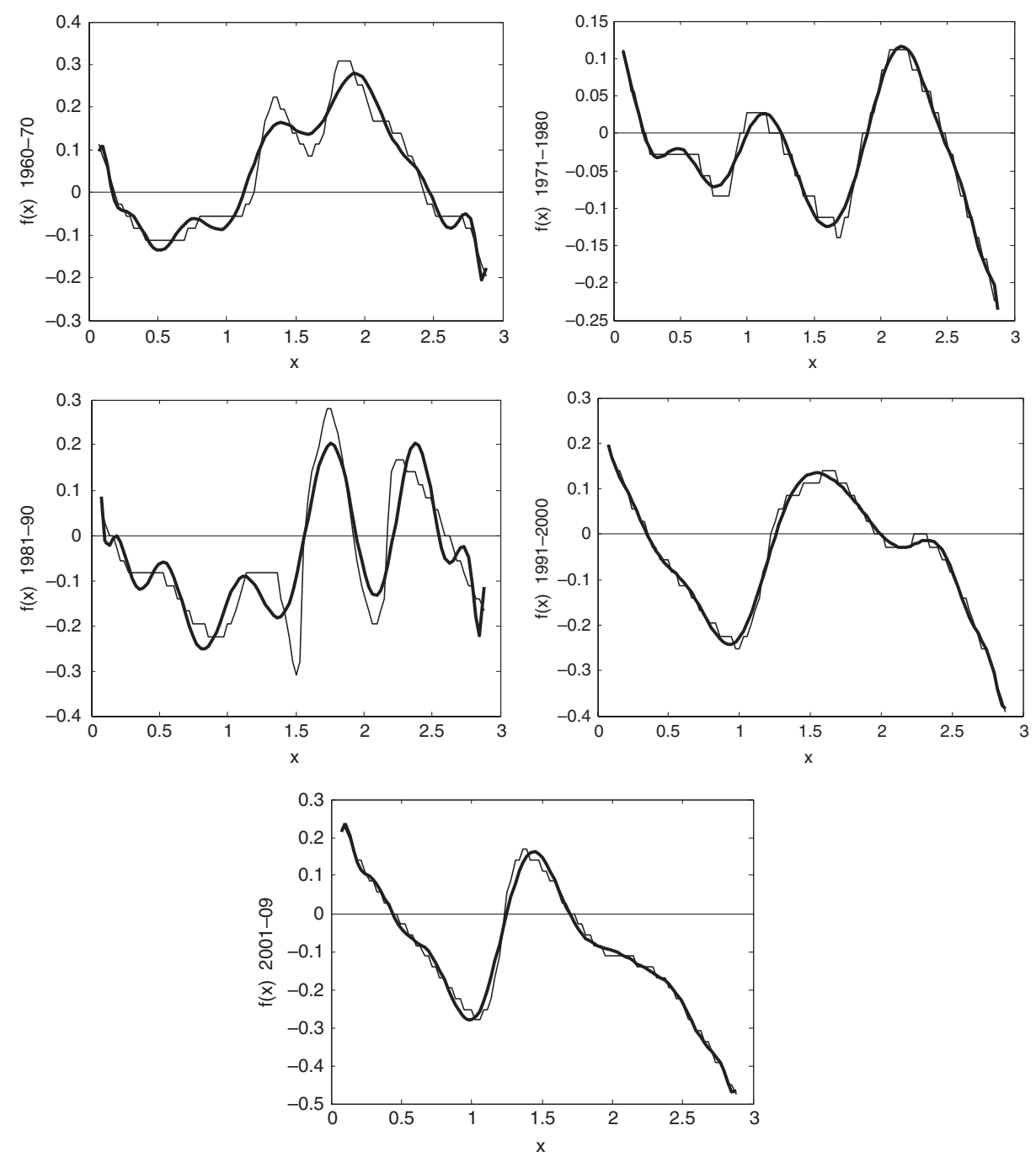

Figure 3. Kolmogorov Density Estimated $f($.$) and 15th-Order Polynomial; Respectively, from Top$ Left, 1960-70, 1971-80, 1981-90, 1991-2000, and 2001-09

Two Latin American countries (Argentina and Venezuela) peaked in the 1960s and 70s and then declined in relative terms as the Asian tigers joined the rich club in the 1990s. China and India, which have been massive laggards in absolute terms, are quickly catching up with the remnants of a middle-income class. However, for other countries that aim to catch up with the Asian tigers, the question of existence of a stable middle-income group becomes important, as we shall see in Section 5, because it eventually reduces the gap between poor and rich and increases the probability of escaping the poverty trap. 

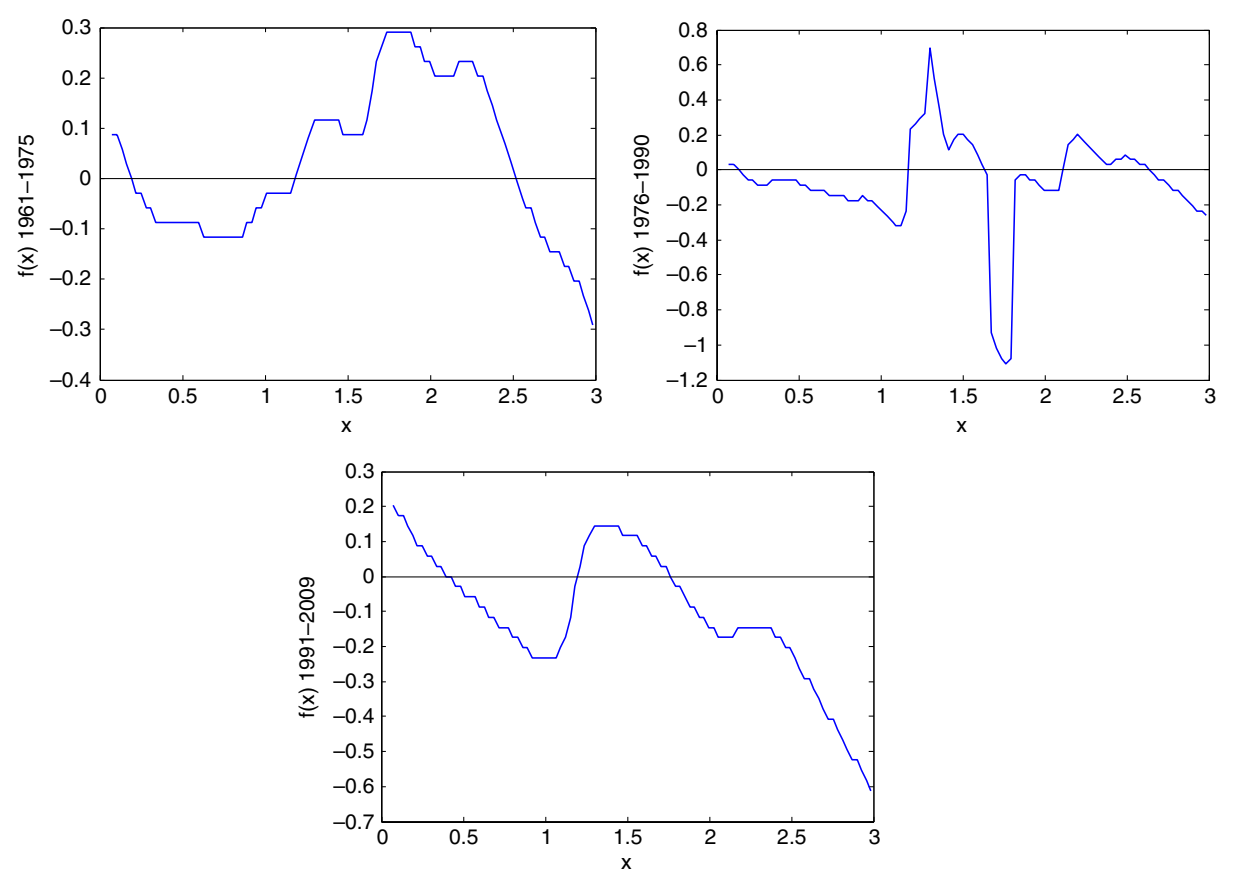

Figure 4. Kernel-Density Estimated $f($.$) ; Respectively, from Top, 1961-75, 1976-91, and 1991-2009$

TABLE 1

Some Countries Getting Relatively Richer, Others Not

\begin{tabular}{|c|c|c|c|c|c|c|c|c|c|c|}
\hline \multirow[b]{2}{*}{ Country } & \multicolumn{2}{|c|}{ 1960-70 } & \multicolumn{2}{|c|}{ 1971-80 } & \multicolumn{2}{|c|}{ 1981-90 } & \multicolumn{2}{|c|}{ 1991-2000 } & \multicolumn{2}{|c|}{ 2001-09 } \\
\hline & Rnk & Clstr & Rnk & Clstr & Rnk & Clstr & Rnk & Clstr & Rnk & Clstr \\
\hline Argentina & 65 & 4 & 77 & 5 & 73 & 4 & 74 & 4 & 70 & 4 \\
\hline Venezuela & 83 & 5 & 80 & 5 & 75 & 4 & 73 & 4 & 64 & 4 \\
\hline S Korea & 50 & 3 & 59 & 4 & 74 & 4 & 79 & 5 & 81 & 5 \\
\hline Taiwan & 54 & 4 & 68 & 4 & 78 & 5 & 82 & 5 & 87 & 5 \\
\hline Singapore & 74 & 4 & 82 & 5 & 89 & 5 & 105 & 5 & 107 & 5 \\
\hline Hong Kong & 75 & 4 & 79 & 5 & 88 & 5 & 93 & 5 & 94 & 5 \\
\hline China & 4 & 2 & 7 & 2 & 26 & 2 & 41 & 3 & 50 & 3 \\
\hline India & 19 & 3 & 23 & 3 & 29 & 2 & 33 & 3 & 36 & 3 \\
\hline
\end{tabular}

Figures 3-4 have confirmed our two stylized facts: (i) stable middle income groups seem to emerge every now and then; and (ii) distribution dynamics change every so often, to destabilize those middle-income groups. To ensure comparability with methods used in the literature, we provide further confirmation in Appendix A using slight modifications of the methods of Bianchi (1997) and Kremer et al. (2001) - to allow for multimodality in the former and continuous state space in the latter, and using our larger data sample. The tests for multimodality in Appendix A confirm the emergence and then disappearance of a middle-income group. We now turn to deriving a formal test using our measure of stochastic stability. 


\section{Stochastic Stability and the Convergence Club Hypothesis}

We consider a Markov process on relative incomes, defined by an estimated transition function $P_{n}(x, A)$ from any point $x$ to any set $A$, with density $t_{n}(x, y) .{ }^{4} \mathrm{We}$ shall define our stochastic stability conditions in terms of the properties of this transition density. We estimate the transition density over a compact set $X$ via the kernel density estimator: ${ }^{5}$

$$
t_{n}\left(x, x^{\prime}\right)=q_{n}\left(x, x^{\prime}\right) / p_{n}(x)
$$

where

$$
p_{n}(x)=\frac{1}{n h} \sum_{i=1}^{n} K\left(\frac{x-x_{i}}{h}\right),
$$

and

$$
q_{n}\left(x, x^{\prime}\right)=\frac{1}{n h} \sum_{i=1}^{n-1} K\left(\frac{x-x_{i}}{h^{1 / 2}}\right) K\left(\frac{x^{\prime}-x_{i+1}}{h^{1 / 2}}\right) .
$$

The last two terms are the corresponding non-parametric estimates of the marginal and joint densities, respectively. We then obtain our estimate of the conditional CDF of this transition as follows:

$$
G_{n}(z \mid x)=\int_{-\infty}^{z} t_{n}\left(d x^{\prime} \mid x\right)
$$

Let the $\alpha$-quantile of this $\mathrm{CDF}$ be denoted $\xi_{n}(\alpha, x)$. We assume that the median $\xi_{n}(0.5, x)$, defined as the smallest root of the equation $G_{n}(z \mid x)=0.5$, is unique. We then investigate stochastic stability in terms of the function $f_{n}(x)=\xi_{n}(0.5, x)-x$.

Definition (Stochastic Stability)

A point $x$ is said to be a stochastically stable zero of the function $f_{n}($.$) if:$

(i) $f_{n}(x)=\xi_{n}(0.5, x)-x=0$,

(ii) $f_{n}\left(x^{\prime}\right)>f_{n}(x)$ for $x^{\prime} \in(x-\varepsilon, x)$ and some $\varepsilon>0$, and

(iii) $f_{n}\left(x^{\prime}\right)<f_{n}(x)$ for $x^{\prime} \in(x, x+\delta)$ and some $\delta>0$.

As noted in Section 2, our analogue of the two-clubs or "twin-peaks" hypothesis of Quah (1997) is thus represented as a function $f($.$) with two stable zeros. The$ classical convergence hypothesis (e.g. as found for within-U.S. income distribution

${ }^{4}$ Formally, for any $x \in \mathbb{R}, P_{n}(x, \cdot)$ is a probability measure on the Borel sigma-algebra, and for a given Borel set $A, P_{n}(., A)$ is a Borel measurable function.

${ }^{5}$ We used Gaussian kernels and likelihood-based time series cross-validation methods for bandwidth selection. The zero points in this and the preceding section are estimated using 10-year lags. For construction of the tests in the following section, we use one-year lags for estimation, and use the averaged standard errors as derived in Appendix B. Upon the suggestion of an anonymous referee, we redid all the analysis using five-year lags and five-year averaging, but none of the results were affected qualitatively (GAUSS ${ }^{\mathrm{TM}}$ codes and tabulated results are available from the authors upon request). 
across states, or within the OECD block, as studied in Johnson, 2000) is represented with a single stable zero. The existence of three stable zeros would add a third alternative to the classical convergence hypothesis and Quah's vanishing middle-income group (or twin clubs) hypothesis. In the remainder of this section, we shall derive the statistical properties of the estimated $f_{n}($.$) , and thus obtain a$ formal test for the number of stable zeros.

One note on transition-kernel regularity assumptions is in order. Separately, Paul Johnson and an anonymous referee have generated examples for mixtures of continuous densities and discrete Markov chains, respectively, where crossing from above of the function $f(a)$ may not mean a stochastic domain of attraction. Both examples utilize multimodal distributions and/or discontinuities in the transition CDF to produce cyclicality (where some countries jump frequently from rich to poor clubs and back). Our estimation method is predicated on the assumption of continuous transition CDF, as discussed in Appendix B. Moreover, nonparametric estimates of densities of growth rates by Maasoumi et al. (2006) were consistently unimodal, suggesting that we need not worry about multimodal transition kernels and the complications that they would introduce. Moreover, the empirical literature has generally found few gradual transitions between clubs (Pittau et al., 2010). With unimodal and smooth transition kernels, multimodal ergodic densities still emerge because of differences in the transition kernel as a function of the current state $x_{t}$, which is what we capture with the function $f($.).

Under the appropriate assumptions summarized in Appendix B, Roussas (1991) proved strong consistency of the estimated CDF $G_{n}(z \mid x)$ (Theorem 2.1 in Roussas, 1991, p. 446) as well as the estimated quantile $\xi_{n}(\alpha, x)$ (Theorem 2.2, ibid). In addition, we can show under suitable assumptions that the estimated $\alpha$-quantile $\xi_{n}(\alpha, x)$ has an asymptotic normal distribution as stated in Proposition 1. We derive the asymptotic variance of the estimated quantile using the techniques in Roussas (1991). The actual asymptotic variance we obtain is a correction for the expression stipulated in Roussas (1969b). Assumptions A.1-A.5 in Appendix B are relatively standard. The crucial assumption is that of stationary-ergodicity/mixing of the true underlying transition kernel. While we question the stationarity of the transition kernel over the entire sample, asserting that different sub-periods were characterized by different transition kernels, we may still maintain that the transition kernel for each sub-period (were it to continue indefinitely) would indeed converge to a unique invariant (ergodic) distribution. When we estimate a transition kernel over a long period, we may think of the object of estimation as a mixture of the various ergodic transition kernels, which can itself be ergodic under appropriate technical assumptions (e.g. Markovian regime switching according to an ergodic probability transition matrix).

Proposition 1. Under suitable assumptions (see Appendix B)

$$
(n h)^{1 / 2}\left[\xi_{n}(\alpha, x)-\xi(\alpha, x)\right] \stackrel{d}{\rightarrow} N\left(0, \tau^{2}(\xi, x)\right),
$$

where

$$
\tau^{2}(\xi, x)=t^{-2}(\xi \mid x) G(\xi \mid x)(1-G(\xi \mid x)) p^{-1}(x) \int K^{2}(z) d z
$$


Replacing the unknown quantities $\{t(\xi \mid x), G(\xi \mid x), p(x)\}$ with their consistent estimates, we can easily perform individual tests for the null hypothesis $H_{0}: \xi(0.5$, $y)=0$. In addition, once we show that the estimates at different points are asymptotically uncorrelated, we can easily construct uniform confidence bands around the function $f($.) based on the point-wise confidence bands. We can also perform simultaneous tests of multiple stable zeros, as shown in Section 4. For now, we conclude this section with two important results: uncorrelatedness of conditional median estimates for the same time period across relative incomes, and across time periods for the same relative income. Those two results will be crucial for performing tests regarding the number of zeros of the estimated $f($.$) .$ Proposition 2 establishes uncorrelatedness of the conditional median estimate at different points for the same period, thus allowing us to construct a simple chi-squared test of multiple-zeros of the function by utilizing only estimated variances at the stipulated zeros. Since our cross-sectional sample is too small to yield sufficiently small estimated variances at the various points, we shall average estimated $f($.$) across five time periods. The calculation of the estimated variance of$ the average is made simple due to Proposition 3, which shows that the estimated conditional median at the same point over different time periods is uncorrelated.

Proposition 2. Under suitable assumptions (see Appendix B)

$$
\operatorname{cov}\left[\left(\xi_{n}(\alpha, x)-\xi(\alpha, x)\right),\left(\xi_{n}(\alpha, y)-\xi(\alpha, y)\right)\right] \stackrel{p}{\rightarrow} 0 \text { for } x \neq y, x, y \in \mathbb{R}
$$

Proposition 3. Under suitable assumptions (see Appendix B)

$$
\operatorname{cov}\left[\left(\xi_{n}^{t+1}(\alpha, x)-\xi^{t+1}(\alpha, x)\right),\left(\xi_{n}^{t}(\alpha, x)-\xi^{t}(\alpha, x)\right)\right] \stackrel{p}{\rightarrow} 0 \text { for } x \in \mathbb{R}
$$

\section{Hypothesis-Testing Support for Stylized Facts}

Before using our measure of stochastic stability to test the hypothesis of existence of a stable middle-income group of countries in various sub-periods of the sample, we present two test results to support the hypothesis of nonstationarity of income dynamics. Results of the first test are shown in Figure 5. This figure reports the results of Kolmogorov-Smirnov tests of equality of the conditional transition CDFs for each initial relative income level for the first and last periods of our sample: $1960-70$ and 2001-09, respectively. ${ }^{6}$ As we can see, the p-values for this test are extremely small for almost all initial relative incomes, with the exception of a small and relatively unpopulated band of relative incomes.

${ }^{6}$ The asymptotic distribution of the Kolmogorov-Smirnov tests is based on the asymptotic normality result of Roussas (1991, Theorem 2.3, p. 447) and the assumption that covariances between the two estimated CDFs for the early 1960s and 2000s are negligible under the maintained hypothesis of an overall mixing data generating process. 


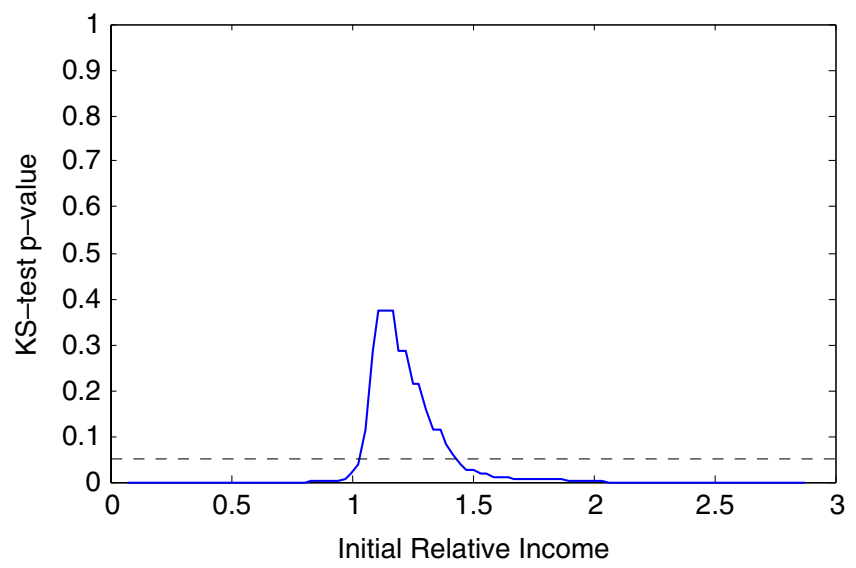

Figure 5. Kolmogorov-Smirnov Test p-values for Equality of conditional CDFs $F\left(x_{t+1} \mid x_{t}\right)$ for 1960-70 vs. 2001-09 at Various Levels of Initial Relative Income $x_{t}$

Consequently, we can conclude that for almost all initial relative incomes, the transition dynamics of countries with such relative incomes were significantly different in the different parts of our sample. Moreover, the initial relative income levels at which the test fails to reject equality of dynamics at the 5 percent level are 14 countries, a very small fraction of our sample.

For comparison with Kremer et al. (2001) and other results in the literature, which focused on limiting distributions, we perform a second KolmogorovSmirnov test of equality of the estimated limiting income distributions of the estimated ten year transitions for the first and last ten-year periods in our sample: 1960-70 and 2001-09, respectively. The pdfs of those limiting distributions are shown in Figure 6. As Kremer et al. (2001) and Quah (1997) have noted, direct calculation of the ergodic distribution through iteration of the transition kernel would be inconsistent. Throughout this paper, we utilize the method of Fiaschi and Romanelli (2009) to renormalize the invariant ergodic density appropriately.

The difference between the two limiting distributions in Figure 6 is obvious, and it results in a p-value of 0.081 for the Kolmogorov-Smirnov test of the equality of the two limiting CDFs. Figures 5-6, along with the test results in Appendix A, further suggest that income distribution dynamics in our sample must be studied separately for the different sub-periods. In particular, we shall focus on the existence of a stochastically stable middle income group in various sub-periods. The existence of such a stochastically stable middle income group influences the probabilities of escape from or falling into a low-income poverty trap, as we shall see in Section 5. First, we turn in the remainder of this section to the formal tests of existence of a stochastically stable middle-income group in various sub-periods.

Utilizing the consistency and asymptotic normality results of Section 3, we can test formally for the existence of multiple stochastically stable income groups 


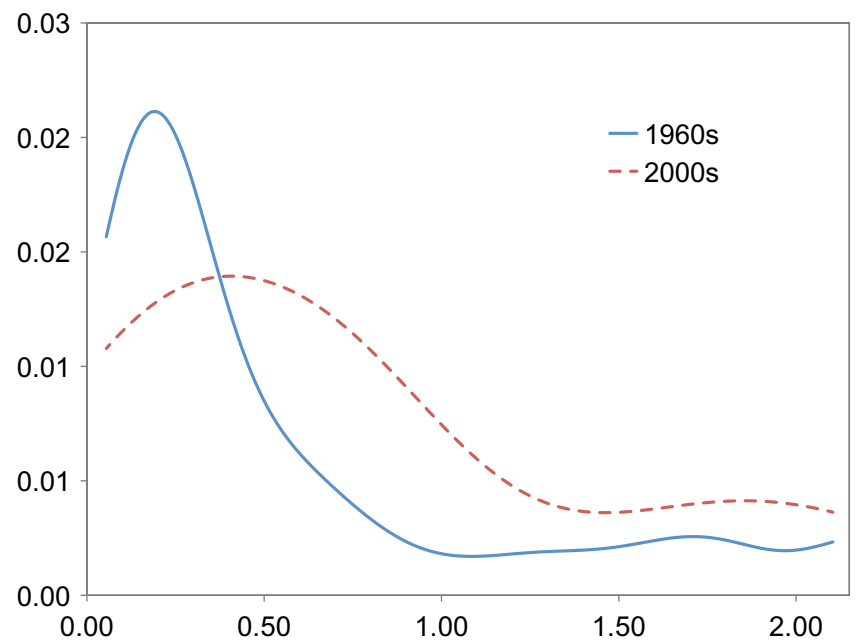

Figure 6. Limiting (Invariant) pdfs for Transitions 1960-70 and 2001-09. Kolmogorov-Smirnov Test for Equality of Limiting CDFs Yields a p-value of 0.08087587

over various time-periods/epochs. Formally, our test will be constructed as follows:

$$
\begin{gathered}
H_{0}:\left.\left(\xi_{n}(0.5, x)-x\right)\right|_{c_{1}}=0 \text { and } \ldots \text { and }\left.\left(\xi_{n}(0.5, x)-x\right)\right|_{c_{l}}=0, \\
H_{1}:\left.\left(\xi_{n}(0.5, x)-x\right)\right|_{c_{1}} \neq 0 \text { or } \ldots \text { or }\left.\left(\xi_{n}(0.5, x)-x\right)\right|_{c_{l}} \neq 0,
\end{gathered}
$$

where $\xi_{n}(0.5, x)$ is an estimated conditional median of income at $t+1$, and $c_{s}, s=1$, $2, \ldots, l$, is the list of points at which our function is equal to zero under the null hypothesis. The five points at which the tests were conducted were chosen for each ten-year period based on the zeros of the function for one-year transitions. For instance, to generate three stable zeros of our function $f_{n}(x)=\xi_{n}(0.5, x)-x$, we would need five zeros of the function, as shown in Figure 2. If we have only two stable zeros (our dynamic version of the twin-peaks hypothesis), then we should reject the null hypothesis of five zeros, in favor of only three zeros (two of which would be stable: representing the rich and poor clubs) or less. Given our interest in testing the three-clubs hypothesis (including a middle-income group), we conducted the test at 5 candidate zeros of our function. If we were interested only in the two-clubs vs. global convergence hypotheses, we would have tested the null of three zeros vs. the alternative of only one. The stylized facts explored in Section 2 prompted us to conduct the formal test for 5 zeros, three of which would be stable.

Since the quantile estimator $\xi_{n}(\alpha, x)$ is asymptotically normally distributed, with the covariance matrix derived in Section 3, we can construct our test statistic as a quadratic form with an asymptotic $\chi^{2}$ distribution under the null hypothesis. Formally, our test statistic is: 


$$
\begin{aligned}
& \left(\xi_{n}\left(0.5, c_{1}\right)-c_{1}, \cdots, \xi_{n}\left(0.5, c_{l}\right)-c_{l}\right)\left(\begin{array}{ccc}
\tau_{1}^{2} & \cdots & 0 \\
\vdots & \ddots & \vdots \\
0 & \cdots & \tau_{l}^{2}
\end{array}\right)^{-1}\left(\begin{array}{c}
\xi_{n}\left(0.5, c_{1}\right)-c_{1} \\
\vdots \\
\xi_{n}\left(0.5, c_{l}\right)-c_{l}
\end{array}\right) \\
& \quad=\sum_{s=1}^{l}\left\{\frac{\xi_{n}\left(0.5, c_{s}\right)-c_{s}}{\tau_{s}}\right\}^{2} \stackrel{d}{\rightarrow} \chi^{2}(l), \text { under } H_{0} .
\end{aligned}
$$

In application, the standard errors $\tau_{s}$ for annual transition estimates were too large. Consequently, we utilized the result in Proposition 3, to average estimates over ten-year periods, thus obtaining reasonably small confidence intervals on $\xi_{n}(0.5, c)-c$. Thus, we conducted the test for three stable relative income clubs (by testing for five zeros of the function $f(a)=\operatorname{median}\left(x_{t+1} \mid x_{t}=a\right)-a$ for the five decades in our sample, corresponding to the plots of the $f($.) functions in Figure 3 ). Table 3 shows the estimated median $\left(x_{t+1} \mid x_{t}=a\right)$ at the listed five points where the function in Figure 3 is at or near zero, as well as the standard error of that estimated median. The $\chi_{5}^{2}$ statistic for our test of five zeros for each decade is listed in the caption for each decade, with *** denoting significance at the 1 percent level (statistic exceeding 15.09; the 5 percent level is 11.07 , and the 10 percent level is 9.24). The existence of three stable zeros is consistent with the results of Akin and Kose (2008), who labeled the three groups as the (rich) North, the (middle-income) Emerging South, and the (poor) Developing South. For completeness, Table 2 also shows similar tests for (at least) two stable zeros (the classical twin-peaks hypothesis), which are of course consistent with the results of Table 3.

TABLE 2

\begin{tabular}{|c|c|c|}
\hline Median & Zero Point & S.E. (median) \\
\hline \multicolumn{3}{|c|}{$1960-70$ (test $\left.\chi^{2}=22.2157^{* * *}\right)$} \\
\hline 0.2010 & 0.2151 & 0.0284 \\
\hline 1.4746 & 1.6726 & 0.0421 \\
\hline 2.4099 & 2.4295 & 0.0434 \\
\hline \multicolumn{3}{|c|}{$1970-80$ (test $\left.\chi^{2}=4.0009\right)$} \\
\hline 0.1842 & 0.1590 & 0.0346 \\
\hline 1.2242 & 1.3083 & 0.0471 \\
\hline 2.4659 & 2.4855 & 0.0372 \\
\hline \multicolumn{3}{|c|}{$1980-90\left(\right.$ test $\left.\chi^{2}=5.5169\right)$} \\
\hline 0.1506 & 0.1590 & 0.0199 \\
\hline 1.8913 & 1.8128 & 0.0402 \\
\hline 2.5612 & 2.5136 & 0.0386 \\
\hline \multicolumn{3}{|c|}{$1990-2000$ (test $\left.\chi^{2}=3.7518\right)$} \\
\hline 0.3468 & 0.3272 & 0.0702 \\
\hline 1.9193 & 1.8128 & 0.0729 \\
\hline 2.3566 & 2.4295 & 0.0587 \\
\hline \multicolumn{3}{|c|}{$2000-09$ (test $\left.\chi^{2}=11.0289^{* * *}\right)$} \\
\hline 0.4455 & 0.5234 & 0.0853 \\
\hline 1.7443 & 1.5886 & 0.0779 \\
\hline 2.2301 & 1.8969 & 0.1339 \\
\hline
\end{tabular}

Tests for Two Stable Clubs by Decade

***Significant at the $1 \%$ level. 
TABLE 3

Tests for Three Stable Clubs by Decade

\begin{tabular}{|c|c|c|}
\hline Median & Zero Point & S.E. (median) \\
\hline \multicolumn{3}{|c|}{$1960-70$ (test $\left.\chi^{2}=101.1376^{* * *}\right)$} \\
\hline 0.2010 & 0.2151 & 0.0284 \\
\hline 1.1317 & 1.4484 & 0.0487 \\
\hline 1.4764 & 1.6726 & 0.0421 \\
\hline 2.2585 & 2.0373 & 0.0434 \\
\hline 2.4099 & 2.4295 & 0.0434 \\
\hline \multicolumn{3}{|c|}{$1970-80$ (test $\left.\chi^{2}=5.1913\right)$} \\
\hline 0.1842 & $0.1590^{n}$ & 0.0346 \\
\hline 0.9158 & 0.9719 & 0.0514 \\
\hline 1.2242 & 1.3083 & 0.0471 \\
\hline 1.9249 & 1.9249 & 0.0217 \\
\hline 2.4659 & 2.4855 & 0.0372 \\
\hline \multicolumn{3}{|c|}{$1980-90$ (test $\left.\chi^{2}=7.4856\right)$} \\
\hline 0.1506 & 0.1590 & 0.0199 \\
\hline 1.3195 & 1.3923 & 0.0520 \\
\hline 1.8913 & 1.8128 & 0.0402 \\
\hline 2.1744 & 2.1772 & 0.0408 \\
\hline 2.5612 & 2.5136 & 0.0386 \\
\hline \multicolumn{3}{|c|}{$1990-2000\left(\right.$ test $\left.\chi^{2}=5.6890\right)$} \\
\hline 0.3468 & 0.3272 & 0.0702 \\
\hline 1.1513 & 1.2802 & 0.1418 \\
\hline 1.9193 & 1.8128 & 0.0729 \\
\hline 2.2557 & 2.1772 & 0.0745 \\
\hline 2.3566 & 2.4295 & 0.0587 \\
\hline \multicolumn{3}{|c|}{$2000-09$ (test $\left.\chi^{2}=15.5880^{* * *}\right)$} \\
\hline 0.4455 & 0.5234 & 0.0853 \\
\hline 1.0622 & 1.4204 & 0.2154 \\
\hline 1.7443 & 1.5886 & 0.0779 \\
\hline 1.9623 & 1.8408 & 0.0907 \\
\hline 2.2301 & 1.8969 & 0.1339 \\
\hline
\end{tabular}

*** Significant at the $1 \%$ level.

Those formal test results support the stylized facts of Section 2, which were based on graphical inspection of our measure of stability of various income groups. They also suggest that framing the problem in terms of "convergence"type hypotheses of any kind may obscure some of the most interesting dynamics. In particular, it would be very useful to model the changes in stochastic transitions themselves, which give rise to the periodic appearance and disappearance of a stable middle-income group. For example, casual ocular inspection of Figure 7, which shows all the zeros of the function $f_{n}($.) estimated for each 1-year transition, suggests that a middle-income group tends to emerge as the gap between the rich and poor clubs gets wider, and to disappear as this gap shrinks in later periods. However, modeling those higher-order dynamics would require a different method, and thus remains beyond the scope of this paper.

\section{Economic Implications and Concluding Remarks}

Quah (2001) noted that a major weakness in the distributional dynamics approach to income distribution was in the area of formal statistical testing and 


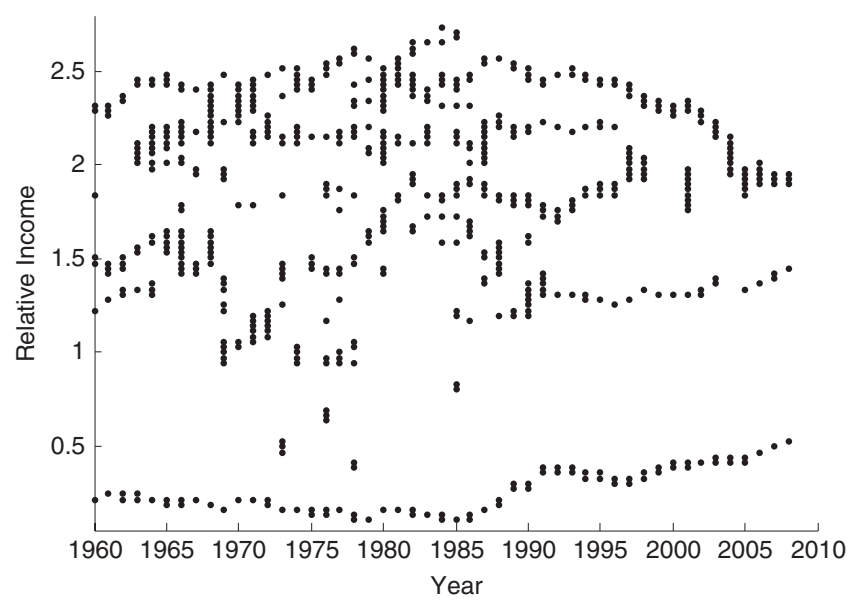

Figure 7. All Zeros of Conditional Median of Annual Transition Less Previous Year's Relative Income

inference. Bianchi (1997) and Kremer et al. (2001) provided valuable early attempts to fill this gap in the literature. However, the first approach remained primarily static (test of multi-modality of estimated cross-country income distribution density at a given point in time), while the second focused only on tests of one versus two modes in the asymptotic (ergodic) distribution, to the exclusion of short-term dynamics. As also noted by Quah (2001), this emphasis on the limiting distributions of estimated probability transition matrices is not sufficient, since classical convergence may only be attainable in this framework after centuries or millennia of increased inequality between the rich and poor.

In contrast, our results have indicated that the probability of escaping poverty, falling into poverty, or converging, has varied over time. Inspecting Figure 7 in conjunction with Figure 3, we can detect a pattern: starting in the 1960s, the gap between the richest and poorest was relatively modest, and we detected no stable middle-income club. The gap between the richest and poorest clubs was growing between 1960 and 1985, and the middle-income club emerged in the middle of this period. Then, from 1986 until the end of our sample, the gap between the richest and poorest clubs shrank again, as some middle-income groups joined the rich club and others joined the poor club, in the process bringing the rich and poor clubs closer together, whereby the middle-income club vanished. The relationship between the size of the gap between rich and poor clubs on the one hand and emergence of middle-income groups on the other was anticipated by Paap and Van Dijk (1998).

Using Chapman-Kolmogorov equations with estimated transition kernels, we plot in Figure 8, respectively, the probabilities of transition (i) from a poor country to a middle-income country, (ii) from a rich to middle-income country, (iii) from middle-income to poor country, and (iv) from middle-income to rich country. This grouping by transitions to middle income (top two panels of Figure 8) and from middle-income (bottom two panels of Figure 8) illustrates the significance of having a stable middle group, and reveals some of the subtleties in observed dynamics. 

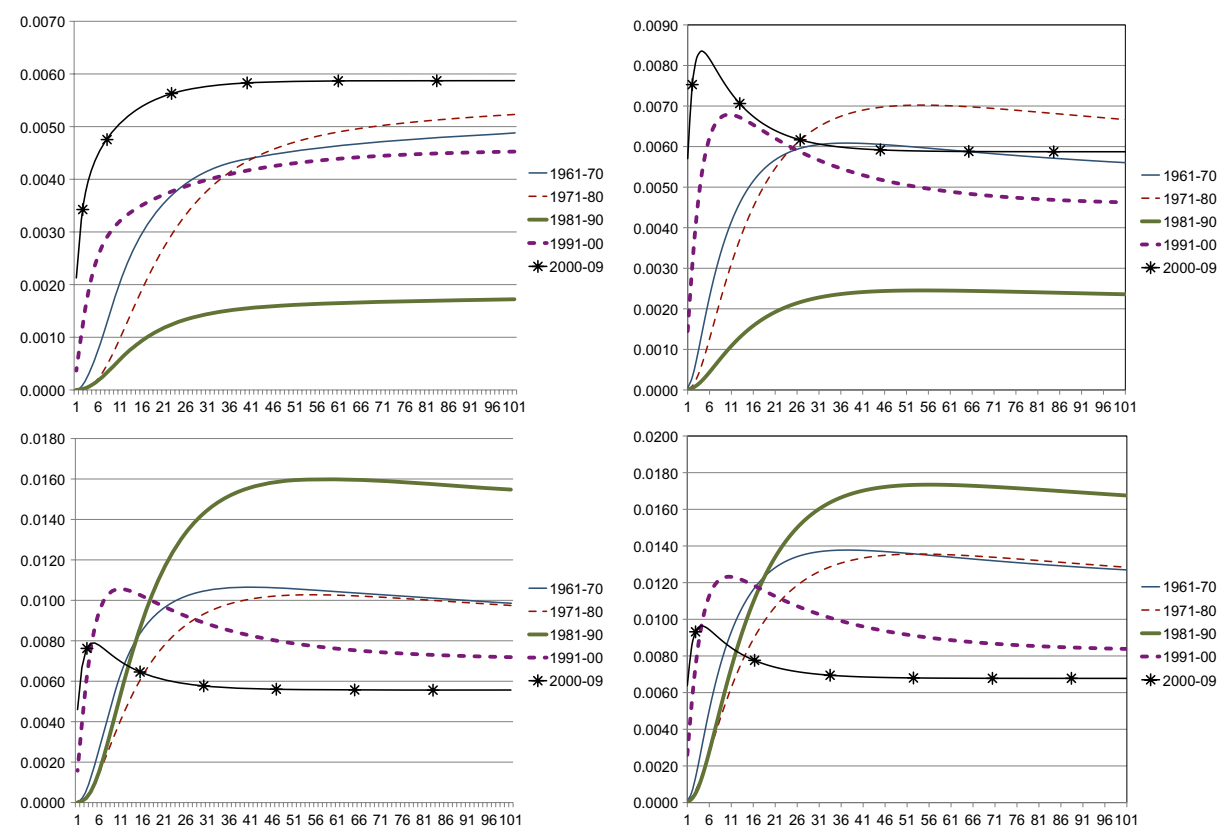

Figure 8. Probabilities of Transition from Relative Income in $n$ Years Rank 5 (very poor) to Rank 50 (middle income) Top Left; Rank 90 (very rich) to Rank 50 (middle income) Top Right; Rank 50 (middle income) to Rank 5 (very poor) Bottom Left; Rank 50 (middle income) to Rank 90 (very rich) Bottom Right

The bottom two panels of Figure 8 are remarkably similar, showing that transition probabilities from middle-income groups to either the rich or poor clubs followed identical patterns by decade, with slightly higher probabilities of getting richer than poorer. The 1980s was a period of hollowing of the middle, as some countries (e.g. Asian tigers) and others (e.g. in Latin America) converged to different clubs. It is therefore not surprising that the early distribution dynamics literature in the 1990s was supportive of the convergence clubs hypothesis. During the 1990s and 2000s, however, the probability of falling precipitously toward the poor club or rising spectacularly toward the rich club declined significantly, as the middle club vanished but contributed, in the process, to narrowing the gap between the rich and poor clubs.

The top two panels of Figure 8 tell a slightly more nuanced, but still optimistic, story. Even though the 1980s were a period of great dynamism for countries in the middle-income group (especially in Southeast Asia and Latin America), it was a period of relative income stability for rich and poor countries. Both groups were much less likely to trend toward middle income during that decade.

As the stable middle-income group vanished, giving way to a narrower richpoor gap, the undeniably good pattern in the top two panels of Figure 8 is that the probability of rising from poverty has increased recently, even as the probability of falling from rich to middle-income status has declined in the long run (after rising briefly over its levels in 1960-80). This is good news, indeed, and supportive of the resurgent evidence for convergence using distribution dynamics as well as traditional regression methods. 
However, as we have seen in this paper, non-stationarity of the dynamics dictates that we do not rush to any conclusions based on extrapolation from current trends. If the higher-order-dynamics cycle continues as observed post World War II, we may very well get another episode of growing rich-poor relative income gaps, followed by the emergence of another stable middle-income group which helps to narrow the gap again, and so on.

Fully understanding the causal underpinnings of this cyclical pattern, and the economic conditions that are conducive for the appearance of a stable middleincome group as the gap widens, eventually causing the gap to shrink, requires deeper economic modeling of the higher level dynamics, which is beyond the scope of this paper. However, the results of this paper suggest that understanding those higher level dynamics may be more fruitful than defending globalization on the basis of "convergence" or condemning it on the basis of "divergence," both notions being asymptotic in nature and ignoring the fundamental and rich nonstationarity of relative income distribution dynamics.

\section{REFERENCES}

Akin, C. and M. Kose, "Changing Nature of North-South Linkages: Stylized Facts and Explanations," Journal of Asian Economics, 19, 1-28, 2008.

Anderson, G., "Toward an Empirical Analysis of Polarization," Journal of Econometrics, 122, 1-26, 2004a.

— , "Making Inferences about The Polarization, Welfare, and Poverty of Nations: A Study of 101 Countries from 1970-1995," Journal of Applied Econometrics, 19, 537-50, 2004b.

Azariadis, C. and A. Drazen, "Threshold Externalities in Economic Development," Quarterly Journal of Economics, 105, 501-26, 1990.

Barro, R. and X. Sala-i-Martin, "Convergence," Journal of Political Economy, 100, 223-51, 1992.

$\longrightarrow$, Economic Growth, McGraw-Hill, New York, 1995.

Bianchi, M., "Testing for Convergence: Evidence from Nonparametric Multimodality Tests," Journal of Applied Econometrics, 12, 393-409, 1997.

Bose, C., "On the Existence and Approximation of Invariant Densities for Nonsingular Transformations on $\mathbb{R}^{d}$," Journal of Approximation Theory, 79, 260-70, 1994.

Bourguignon, F. and C. Morrison, "Inequality among World Citizens: 1820-1992," American Economic Review, 92, 727-44, 2002.

Bulli, S., "Distribution Dynamics and Cross-Country Convergence: A New Approach," Scottish Journal of Political Economy, 48, 226-243, 2001.

Desdoigts, A., "Patterns of Economic Development and the Formation of Clubs," Journal of Economic Growth, 4, 305-30, 1999.

Durlauf, S., "Nonergodic Economic Growth," Review of Economic Studies, 60, 349-66, 1993.

Durlauf, S., P. Johnson, and J. Temple, "Growth Econometrics," in P. Aghion and S. Durlauf (eds), Handbook of Economic Growth, Vol. 1A, North Holland, Amsterdam, 555-677, 2005.

Fiaschi, D. and A. Lavezzi, "Distribution Dynamics and Nonlinear Growth," Journal of Economic Growth, 8, 379-401, 2003.

Fiaschi, D. and D. Romanelli, "Nonlinear Dynamics in Welfare and the Evolution of World Inequality," Banca D'Italia Working Paper \#724, October 2009.

Fischer, S., "Globalization and Its Challenges," American Economic Review, 93, 1-30, 2003.

Johnson, P., "A Nonparametric Analysis of Income Convergence across the US States," Economics Letters, 69, 219-23, 2000.

- "A Continuous State Space Approach to Convergence by Parts," Economics Letters, 86, 317-21, 2005.

Kremer, M., A. Onastski, and J. Stock, "Searching for Prosperity," Carnegie-Rochester Conference Series on Public Policy, 55, 275-303, 2001.

Li., T., "Finite Approximation of Frobenius-Perron Operators: A Solution to Ulam's Conjecture," Journal of Approximation Theory, 17, 177-86, 1976.

Maasoumi, E., J. Racine, and T. Stengos, "Growth and Convergence: A Profile of Distribution Dynamics and Mobility," Journal of Econometrics, 136, 483-508, 2006. 
Mankiw, N., D. Romer, and D. Weil, "A Contribution to the Empirics of Economic Growth," Quarterly Journal of Economics, 107, 407-37, 1992.

Paap, R. and H. van Dijk, "Distribution and Mobility of Wealth of Nations," European Economic Review, 42, 1269-93, 1998.

Phillips, P. and D. Sul, "The Elusive Empirical Shadow of Growth Convergence," Cowles Foundation Discussion Paper, No. 1398, 2003.

Pittau, M. and R. Zelli, "Empirical Evidence of Income Dynamics across EU Regions," Journal of Applied Econometrics, 21, 605-28, 2006.

Pittau, M., R. Zelli, and P. Johnson, "Mixture Models, Convergence Clubs, and Polarization," Review of Income and Wealth, 56, 102-22, 2010.

Quah, D., "Empirical Cross-Section Dynamics in Economic Growth," European Economic Review, 37, 426-34, 1993a.

, "Galton's Fallacy and Tests of the Convergence Hypothesis," Scandinavian Journal of Economics, 95, 427-43, 1993b. 1995.

, "Convergence Empirics across Economies with (Some) Capital Mobility," Journal of Economic Growth, 1, 95-124, 1996a.

, "Twin Peaks: Growth and Convergence in Models of Distribution Dynamics," Economic Journal, 106, 1045-55, 1996b.

— "Empirics for Growth and Distribution: Polarization, Stratification, and Convergence Club," Journal of Economic Growth, 2, 27-59, 1997.

- "Searching for Prosperity: A Comment," Carnegie-Rochester Conference Series on Public Policy, 55, 305-19, 2001.

Roussas, G., "Nonparametric Estimation in Markov Processes," Annals of the Institute of Statistical Mathematics, 21, 73-87, 1969a.

- "Nonparametric Estimation of the transition Distribution Function of a Markov Process," Annals of Mathematical Statistics, 40, 1386-400, $1969 \mathrm{~b}$.

- "Estimation of Transition Distribution Function and Its Quantiles in Markov Processes: Strong Consistency and Asymptotic Normality," in G. Roussas (ed.), Nonparametric Functional Estimation and Related Topics, Kluwer, Dordrecht, 443-62, 1991.

Ryu, D., "Mean Reversion in Macroeconomic and Financial Data," PhD Dissertation, Rice University, 2004.

Sala-i-Martin, X., "The Disturbing 'Rise' of the Global Income Inequality," NBER Working Paper 8904, 2002a. , "The World Distribution of Income (Estimated from Individual Country Distributions)," NBER Working Paper 8933, 2002 b.

Silverman, B., "Using Kernel Density Estimates to Investigate Multi-modality," Journal of the Royal Statistical Society, Series B, 43, 97-99, 1981.

Vollmer, S., H. Holzmann, and F. Schwaiger, "Peaks vs. Components," Review of Development Economics, forthcoming, 2012.

\section{SUPPORTING INFORMATION}

Additional Supporting Information may be found in the online version of this article:

Appendix A: Modified Bianchi (1997) and Kremer, Onastski, and Stock (KOS) (2001) Tests: More Support for Non-Stationarity

Table A1: Static Multimodality Test using Bianchi's (1997) Method

Table A2: Dynamic Multimodality Test of Limiting Density

Figure A1: From Top Left to Bottom Right, Respectively: Estimated Densities Imposing Unimodality, Bimoaality, Trimodality, and Quatrimodality

Appendix B: Asymptotic Distribution of Test Statistic 